\title{
Politicas de salud en áreas de frontera. El caso de Argentina y Paraguay
}

\author{
Health policy in border areas. The case of Argentina and Paraguay
}

\section{Maria Alejandra Fantin ${ }^{1}$ y Blanca Elizabeth Ponce $^{2}$}

Resumen: En materia de salud, la frontera entre Argentina y Paraguay posee una intensa historia de cooperación internacional que se ha incrementado en los últimos años como consecuencia de la aparición de enfermedades emergentes y reemergentes como el cólera, la fiebre amarilla, el dengue y el aumento de la tuberculosis. El presente trabajo tiene como objetivo analizar los convenios establecidos entre ambos paises en relación a la atención de la salud, en particular aquellos de carácter específico referidos a la frontera y los firmados entre jurisdicciones provinciales/departamentales.

Palabras clave: Frontera, salud, politicas, Argentina, Paraguay.

Abstract: In health field, the border between Argentina and Paraguay maintains a strong history of international cooperation which has increased in recent years as a result of the occurrence of emerging and reemerging diseases such as cholera, yellow fever, dengue and the rise in tuberculosis. This paper aims to analyze the agreements signed by both countries in relation to health care, particularly those with specific nature related to the border and signed by provincial/departmental jurisdictions.

Keywords: Border, health, policies, Argentina, Paraguay.

\section{INTRODUCCIÓN}

El desarrollo natural de la enfermedad no reconoce limites ni fronteras, situación que muchas veces puede incidir en la dinámica internacional, originando o reforzando nuevos campos de cooperación o de conflicto en la comunidad internacional afectando de esta manera las relaciones económicas, politicas y sociales entre los estados (Frenk y Chacón, 1992).

Si bien las politicas de la salud son responsabilidad de los estados, el creciente y acelerado proceso de globalización demanda que las políticas y acciones sanitarias locales y nacionales requieran de la cooperación internacional y de

1 Doctora en Demografia. Investigadora del Instituto de Investigaciones Geohistóricas, CONICET-UNNE. (mafantin@gmail com)

2 Doctora en Demografia. Becaria del Instituto de Investigaciones Geohistóricas, CONICET-UNNE. (blancae_ponce@yahoo.com.ar)

Recibido: 23/10/2015; Aceptado: 27/05/2015. http://dx.doi.org/10.18004/riics.2015.diciembre.241-258 
intervenciones colectivas transnacionales para garantizar la salud de las poblaciones, sobre todo ante problemas de la salud y riesgos que trascienden fronteras. En los últimos años, el diálogo entre los campos de la salud y las relaciones internacionales se ha incrementado. Sin duda la salud tiene, en el momento actual, una mayor presencia y prioridad en la agenda internacional, hecho que tiene consecuencias notables y plantea desafios tanto para los países como para las organizaciones internacionales (Organización Panamericana de la Salud-Organización Mundial de la Salud, 2008).

Los temas relacionados con la salud afectan a los países tanto hacia el interior como al exterior de sus límites internacionales, es decir, de la misma manera que no es posible analizar el proceso salud-enfermedad como si tuviera lugar solamente dentro de las fronteras nacionales, es inadmisible interpretar la naturaleza de los problemas de salud en el ámbito internacional sin hacer referencia a sus expresiones nacionales. Ahora bien, como lo expresa Panisset (1992) para alcanzar el objetivo de hallar soluciones en común, no basta con dar la impresión de que el proceso de salud-enfermedad está cada vez más internacionalizado; también hay que internacionalizar las soluciones.

La autonomía de cada Estado demanda el desarrollo de estrategias que permitan asegurarle sus intereses y delimitar sus relaciones con los demás países. La Organización Panamericana de la Salud (OPS) propone cuatro ámbitos de acción para fortalecer las politicas y las relaciones internacionales en materia de salud (Organización Panamericana de la Salud, 2008).

a) Rectoría en salud (qué): capacidad de gestión del estado para implementar y llevar a buen término sus objetivos y politicas nacionales e internacionales en materia de salud con respecto a las relaciones internacionales.

b) Diplomacia sanitaria mundial (para qué): conjunto de funciones para el manejo de las relaciones entre los estados y otros actores relevantes vinculados con la salud, que persiguen la búsqueda de soluciones y acuerdos para el beneficio de la salud de sus pueblos.

c) Gestión de la cooperación internacional (cómo): refiere a la necesidad de capitalizar en beneficio nacional, es decir de toda la población del país, las posibilidades y oportunidades que ofrece la cooperación internacional. 
d) Desarrollo de capacidades nacionales para la acción internacional en la salud (con qué): requiere decisión política, recursos suficientes y una clara estrategia de formación de redes de instituciones, activos procesos de intercambio, así como estrategias e intervenciones para el fortalecimiento institucional.

Dentro de este marco, la cooperación internacional se pueda dar tanto en forma bilateral, cuando se establece mediante acuerdo entre dos países y las respectivas agencias oficiales financieras o técnicas de esos dos países; multilateral cuando la relación es entre uno o varios países y uno o varios organismos internacionales; horizontal cuando sus actores principales son dos o más países en desarrollo y se refiere a las relaciones bilaterales y multilaterales que implican gobiernos, instituciones, corporaciones, personas y organizaciones no gubernamentales de dos o más países en desarrollo, también llamada Cooperación Técnica entre Países (Organización Panamericana de la Salud, 2007).

El presente trabajo tiene como objetivo analizar los convenios establecidos entre Argentina y Paraguay en relación a la atención de la salud, en particular aquellos de carácter específico referidos a la frontera y los firmados entre jurisdicciones provinciales/departamentales.

\section{Evolución de las relaciones fronterizas}

Es común que en la percepción del fenómeno fronterizo se entrecrucen dos nociones a ser tenidas en cuenta: la frontera-línea o border y la frontera-zona o frontier.

La frontera como línea marca el confin de un estado, los límites precisos dentro de los cuales ese Estado ejerce su jurisdicción, por lo tanto tiene una connotación y orientación fundamentalmente jurídica, es perfectamente tangible e inviolable, y su existencia está sujeta a tratados o acuerdos jurídicos o políticos.

La frontera como zona, comprende aquellos territorios contiguos a los límites geográficos del Estado -ya sean municipios, departamentos o localidades- en cuyas actividades sociales, comerciales y económicas se advierte la influencia directa del fenómeno fronterizo. Es un término que posee una acepción más bien social y económica y es definida desde una perspectiva cultural y geográfica como un espacio de convergencia, de hibridez, de yuxtaposición de sujetos, instituciones y prácticas pertenecientes a contextos diferentes. Su existencia es determinada 
fundamentalmente por la presencia de manifestaciones culturales, sociales, económicas, etc.

En este sentido la frontera surge de una relación, de un encuentro, de la convergencia y la interacción de dos sociedades más o menos diferentes, de dos realidades sociales, económicas, culturales, políticas que pueden tener mucho o poco en común, pero que en la "frontera" se unen, se combinan dando una realidad única.

La frontera contiene posibilidades de conflictos, pero también de cooperación y constituye un territorio donde muchas veces las necesidades locales están soslayadas por las necesidades nacionales. Cada frontera ofrece un escenario en el que se combinan desafios y oportunidades y donde se genera un doble proceso cultural con la permanencia de determinados componentes tradicionales de la cultura de origen y la incorporación de elementos y modos de vida de "el otro", en un proceso que es caracterizado, en la actualidad, como "transnacional" (Palau, 1995).

Para los pobladores fronterizos, es indudable que la frontera constituye un lugar de encuentro entre dos naciones, que les permite diseñar una estrategia de vida diferente. El poblador fronterizo "vive de la frontera"; "la nacionalidad es una variable que tiene una importancia secundaria en las zonas de fronteras, ya que en estas áreas de interfase se profundizan los intercambios de manera significativa y es frecuente la doble nacionalidad, procesos de hibridización cultural o de mezcla de nacionalidades" (Palau, 1995, p. 3).

En las fronteras, permeables, dinámicas, donde los intercambios poblacionales son importantes, las diferencias entre los estados nacionales se minimizan, las poblaciones dejan de tener las características propias de su país para adquirir las cualidades de poblador fronterizo con matices propios, producto de la conjunción de las dos sociedades, de las dos culturas.

La relación fronteriza entre Argentina y Paraguay es muy antigua y se remonta a la época colonial. En esta etapa no existía una delimitación establecida entre las jurisdicciones de las ciudades de Asunción y Buenos Aires. La navegación fluvial comunicó ambas poblaciones y vinculó a Corrientes y Paraguay en lo económico, mientras que las Misiones Jesuíticas Guaranies integraron un espacio geográfico común desde inicios del siglo XVII. Las Misiones Jesuíticas Guaranies o Reducciones Jesuíticas Guaraníes fueron un conjunto de treinta 
pueblos misioneros fundados a partir del siglo XVII por la orden religiosa católica de la Compañia de Jesús entre los indios guaraníes y pueblos afines, que tenían como fin su evangelización y que se ubicaron geográficamente -quince- en las actuales provincias de Misiones y Corrientes, en Argentina, -ocho- en el Paraguay y -las siete restantes- en las denominadas Misiones Orientales, situadas al suroeste del Brasil. Otras áreas como el Pilcomayo carecieron de vinculación hasta fines del siglo XIX y fueron el hábitat de naciones indígenas antes y después de la conquista española y de la formación de las nacionalidades (Maeder, 2010).

Los distintos tramos de la frontera han vivido historias diferentes. La región chaqueña o Gran Chaco, ubicada al oeste de los ríos Paraná y Paraguay, se mantuvo durante toda la etapa colonial bajo el dominio de los pueblos indígenas que la habitaban, si bien hubo intentos de poblar la región y de instalar reducciones para los grupos aborigenes, estas no prosperaron. Las dos poblaciones más importantes, Concepción del Bermejo y Esteco fundadas por colonizadores provenientes de Asunción en el primer caso y del Tucumán en el segundo, no prosperaron y fueron abandonadas por sus pobladores y las reducciones jesuíticas que se fundaron en el Chaco en el siglo XVIII fue promovida desde las ciudades vecinas a dicha frontera: San Carlos y Rosario del Timbó desde Asunción; San Fernando desde Corrientes, etc. (Maeder, 2010). A pesar de estos intentos, no parece que se haya planteado formalmente una delimitación de estos territorios entre las provincias de Buenos Aires y Paraguay. Los hechos permiten apreciar que cada ciudad limítrofe ejercía la jurisdicción sobre el territorio que tenía frente a ella (Maeder, 2010).

La frontera del Pilcomayo, definida internacionalmente en 1878, es un área de escaso poblamiento y desarrollo, con ganadería extensiva y hasta hoy, con importantes asentamientos indígenas en ambas márgenes del río. A su vez, el límite fluvial requirió acuerdos binacionales posteriores que definieran su curso hasta el Tratado Complementario de 1945, que estableció el régimen de administración de las aguas. El paulatino retroceso del río por acumulación de sedimentos en los tramos Esmeralda $\left(62^{\circ} 30^{\prime} \mathrm{W}\right)$ y Horqueta $\left(61^{\circ} \mathrm{W}\right)$ han provocado problemas entre los habitantes locales, requerido nuevos contactos diplomáticos y técnicos y la definición de obras que permitan el equitativo aprovechamiento de las aguas en ambas márgenes (Rey Balmaceda, 1979).

El tramo a lo largo del Río Paraguay tuvo una historia más intensa. La margen paraguaya experimentó una ocupación muy anterior a la argentina, con centros urbanos que sirvieron de escala a la navegación fluvial. En la ribera 
occidental, el poblamiento fue posterior a la creación del Territorio Nacional del Chaco en 1872 y sus núcleos más importantes fueron la colonia Formosa (1879), el complejo agroindustrial Las Palmas (1882) y Clorinda como centro de intercambio fronterizo. Desde esos núcleos se fue proyectando la paulatina ocupación del espacio y la diversificación de actividades forestales y agrícolas que atrajeron mano de obra vecina. La traza de caminos modernos y la habilitación de puentes sobre el Pilcomayo y el Paraguay aceleraron dichos intercambios, que se tornaron mucho más fluidos en las últimas décadas.

En cambio, la frontera fluvial del Paraná, entre Corrientes y Posadas, no dio lugar a una relación tan importante. Los vados del río en Paso Patria e Itatí sirvieron desde antiguo para el cruce de arreos de ganado, al tiempo que el límite internacional entre Ituzaingó y Posadas se mantuvo impreciso en la margen argentina, sobre la cual el Paraguay sostuvo guarniciones y tranqueras fronterizas hasta 1865 (Maeder, 2010). Las obras hidroeléctricas de la presa de Yacyretá han modificado la anterior situación de aislamiento que vivía dicha área.

A su vez, el tramo del Alto Paraná, desde Posadas hasta Puerto Iguazú, si bien estuvo parcialmente integrado en la época de las misiones jesuíticas, vivió luego un prolongado aislamiento que concluyó recién a fines del siglo XIX con la creación del Territorio Nacional de Misiones en 1881. La producción de yerba mate y de madera, el tráfico fluvial y posteriormente, la creación de pueblos y colonias en una y otra margen, vitalizaron la relación. Más recientemente, la pavimentación de los caminos, cuando las rutas comenzaron a ser pavimentadas en la década de 1960 en Argentina, y algo más tarde en el Paraguay (Pérez, 1985), el puente Posadas-Encarnación, denominado Puente Internacional San Roque González de Santa Cruz erecto sobre el río Paraná, que comunica a las ciudades de Posadas, en la Argentina, y Encarnación, en el Paraguay inaugurado en 1990, el incremento turístico hacia las Cataratas del Iguazú, abrieron una nueva etapa de relación fronteriza, particularmente conflictiva en la zona de la Triple Frontera entre Puerto Iguazú (Argentina), Ciudad del Este (Paraguay) y Foz de Iguazú (Brasil).

La zona de la Triple Frontera, configura un espacio transnacional marcado por contrastes entre los países que la comparten y al interior de sus regiones, en el cual las asimetrias más notables son la pobreza y la desigualdad. La triple frontera es un área muy peculiar, donde se desarrollan diversos procesos que favorecen la integración regional y deberian ser potenciados, como ser: la actividad comercial; la operación de la represa hidroeléctrica de Itaipú; los estudios del Acuífero Guaraní mientras otros la obstaculizan, y deberian ser controlados como ser: el 
contrabando; la expansión de la producción de sojeros brasileños que operan en un régimen de enclave; el control de la biodiversidad por actores extra regionales (Fogel, 2008).

\section{La salud en el marco de la integración regional}

\section{Mercado Común del Sur, MERCOSUR}

Argentina y Paraguay junto con Uruguay, y Brasil no han querido quedar fuera de la firme tendencia de Europa y el resto del mundo desarrollado a formar espacios económicos ampliados con la finalidad de aprovechar las escalas de producción y de inversión.

De esta manera, el 26 de marzo de 1991 la República Argentina, la República Federativa de Brasil, la República del Paraguay y la República Oriental del Uruguay, suscribieron el Tratado de Asunción, creando el Mercado Común del Sur, MERCOSUR (1991), cuyo objetivo primordial es la integración de los cuatro Estados Partes, a través de la libre circulación de bienes, servicios y factores productivos, el establecimiento de un arancel externo común y la adopción de una política comercial común, la coordinación de políticas macroeconómicas y sectoriales y la armonización de legislaciones en las áreas pertinentes, para lograr el fortalecimiento del proceso de integración (Secretaría del Mercosur, 2012).

Tres años más tarde, en diciembre de 1994, el MERCOSUR fue dotado de personalidad jurídica internacional, con la aprobación del Protocolo Adicional al Tratado de Asunción sobre la Estructura Institucional del Mercosur - el Protocolo de Ouro Preto -, por el cual se establece, además, la estructura institucional del Mercosur (Secretaría del Mercosur, 2012).

A partir de entonces continuaron los avances de integración y actualmente el MERCOSUR cuenta, además de los cuatro miembros plenos iniciales, con la República Bolivariana de Venezuela y el Estado Plurinacional de Bolivia y con seis países asociados: Chile, Colombia, Ecuador, Perú, Guyana y Surinam.

En el ámbito del MERCOSUR existen dos foros de carácter deliberativo que tienen a la Salud como principal objeto de trabajo: El Subgrupo de Trabajo No11Salud (SGT No11). Este subgrupo fue creado en la XXIV Reunión del Grupo Mercado Común (GMC), realizada en la ciudad de Fortaleza, Brasil, entre el 12 al 14 de diciembre de 1996, a través de la Resolución GMC N 151/96. La Resolución 
GMC N ${ }^{\circ}$ 04/98 establece y aprueba las pautas de trabajo de dicho Subgrupo, y las Reuniones de Ministros de Salud creada en el año 1995, a través de la Decisión del Consejo del Mercado Común $\mathrm{N}^{\circ}$ 03/95; ambos foros formalizan propuestas que son elevadas a los Órganos Decisorios del MERCOSUR.

El SGT No11-Salud fue creado como órgano técnico de carácter deliberativo que centraliza el tratamiento de las cuestiones referidas a la armonización de las legislaciones nacionales en materia de regulación de la salud y la compatibilización de los sistemas de control sanitario entre los Estados Partes.

Este foro tiene tres comisiones: la primera para la armonización de las normativas vinculadas a productos para la salud; la segunda de vigilancia en salud, plantea la necesidad de vigilar y dar normas para enfrentar cualquier tipo de amenaza vinculada a la posible transmisión de enfermedades y que cuenta con una sub comisión que tiene que ver con el control sanitario de puertos, aeropuertos, terminales y puntos de frontera; y finalmente, la tercera de prestación de servicios de atención de salud, tiene como objetivo garantizar patrones similares de calidad en la atención de salud en los estados parte (Tobar, 2009; Secretaría Administrativa del Mercosur, 2001).

Dentro de la Reunión de Ministros de la Salud, existen diferentes subgrupos llamados "Núcleos de Articulación": a) Sistema de Información y Comunicación en Salud; b) Salud de Fronteras; c) Atención Médica y Uso Racional de la Tecnología y d) Cooperación Internacional en Salud.

El subgrupo salud de fronteras, que es el que nos interesa particularmente en ésta oportunidad, apoya y estimula la elaboración de acuerdos bilaterales de salud en las fronteras considerando sus especificidades (Tobar, 2011; Secretaría Administrativa del Mercosur, 2001).

Asimismo, el 28 de noviembre de 2008, se ha creado el Observatorio MERCOSUR de Sistemas de Salud (OMSS) por Acuerdo $\mathrm{N}^{\circ} 18 / 08$ de la XXV Reunión de Ministros de Salud del MERCOSUR realizada en Río de Janeiro, con el objetivo de producir, diseminar e intercambiar conocimientos sobre los sistemas de salud de los Estados Partes, mediante la sistematización y análisis de los aspectos vinculados al abordaje de los determinantes sociales de la salud, los modelos de atención, financiamiento y gestión para apoyar los procesos de formulación de políticas y toma de decisiones en materia de salud priorizadas en el ámbito del MERCOSUR (Observatorio Mercosur de Sistemas de Salud, 2008). 


\section{Unión de Naciones Suramericanas (UNASUR)}

Otra importante iniciativa de integración sudamericana es la creación de la Unión de Naciones Suramericanas (UNASUR). En el Tratado Constitutivo firmado en Brasilia, el 23 de mayo de 2008 los Jefes de Estado y de Gobierno de los 12 países sudamericanos -Argentina, Bolivia, Brasil, Chile, Colombia, Ecuador, Guyana, Paraguay, Perú, Surinam, Uruguay y Venezuela- afirman su determinación de construir una identidad y ciudadania suramericana y desarrollar un espacio regional integrado en lo político, económico, social, cultural, ambiental, energético y de infraestructura, para contribuir al fortalecimiento de la unidad de América Latina y el Caribe (UNASUR, 2014).

Concretamente, el artículo $2^{\circ}$ del Tratado define el objetivo y por ende el alcance de la organización en estos términos: "La Unión de Naciones Suramericanas tiene como objetivo construir, de manera participativa y consensuada, un espacio de integración y unión en lo cultural, social, económico y político entre sus pueblos, otorgando prioridad al diálogo político, las políticas sociales, la educación, la energia, la infraestructura, el financiamiento y el medio ambiente, entre otros, con miras a eliminar la desigualdad socioeconómica, lograr la inclusión social y la participación ciudadana, fortalecer la democracia y reducir las asimetrías en el marco del fortalecimiento de la soberanía e independencia de los Estados" (UNASUR, 2014, p. 9).

En el marco de UNASUR funciona el Consejo de Salud Suramericano (UNASUR Salud), constituido por los Ministros de Salud como órgano de consulta y consenso en temas de salud. El Consejo de Salud Suramericano fue creado en la ciudad de Río de Janeiro, Brasil, el 28 de noviembre de 2008.

El Consejo tiene como objetivo general consolidar Suramérica como un espacio de integración en Salud, que contribuya a la salud para todos y al desarrollo, incorporando e integrando los esfuerzos y logros subregionales de MERCOSUR, ORAS CONHU y OTCA.

Entre los objetivos específicos se puede resaltar: Privilegiar las acciones de UNASUR Salud en el ámbito de las fronteras; identificar determinantes sociales críticos para la Salud y propiciar politicas y acciones intersectoriales; fortalecimiento de los ministerios de Salud; priorizar las acciones de UNASUR Salud en poblaciones vulnerables y excluidas, asimismo en áreas geográficas en alto riesgo, entre otras (UNASUR, 2008). 


\section{Acuerdos bilaterales}

En materia de salud, la frontera entre Argentina y Paraguay posee una intensa historia de cooperación internacional que se ha incrementado en los últimos años como consecuencia de la aparición de enfermedades emergentes y reemergentes como el cólera, la fiebre amarilla, el dengue y el aumento de la tuberculosis.

En abril de 1978 se firmó el Convenio Sanitario entre el Gobierno de la República Argentina y el Gobierno de la República del Paraguay, en el cual ambos países reconocieron la existencia de problemas comunes de salud en las zonas limitrofes (Paludismo. Fiebre amarilla, enfermedad de Chagas-Mazza, enfermedades venéreas, lepra, esquistosomiasis, rabia, cólera, otras enfermedades transmisibles y antropozoonosis) "a los efectos de eliminar o atenuar los daños a la salud en las comunidades de las referidas zonas geográficas, es necesario el establecimiento de un marco normativo adecuado que permita la adopción de acciones coordinadas capaces de mejorar las respectivas situaciones sanitarias" (Convenio Sanitario, 1978), y la necesidad de acciones coordinadas capaces de mejorar las respectivas situaciones sanitarias (formación y adiestramiento de recursos humanos, control sanitario de poblaciones migrantes, provisión de drogas antiblásticas a través de un banco de drogas, control de estupefacientes y otras drogas peligrosas, educación para la salud, intercambio de información) ..."es de interés común el fortalecimiento de una cooperación técnica que permita compartir posibilidades y conocimientos en el campo de la salud, tanto a través de programas y actividades específicas como por especialistas de servicios de consultoria, de información" (Convenio Sanitario, 1978).

Dicho acuerdo consta de dieciséis artículos; los nueve primeros establecen las medidas que ambos países convienen realizar a fin de controlar cada una de las patologías que fueron consignadas como comunes. Los siguientes seis hacen referencia a las actividades tendientes a fortalecer la cooperación técnica para la formación de recursos humanos, control sanitario, provisión de drogas etc. El último hace alusión a las disposiciones finales en las que se establece la designación de un comité de coordinación, la duración del acuerdo en 5 años, entre otras normas operativas.

Posteriormente, el 30 de octubre de 1992 se firma el Convenio entre el Gobierno de la República Argentina y el Gobierno de la República del Paraguay en materia de salud fronteriza, y el 28 de noviembre de 1995 su protocolo adicional. 
Es importante destacar que el presente convenio hace explícito en la denominación del mismo el concepto de salud fronteriza, como así también en todos los considerandos. Contiene diez artículos, en el primero se exponen las acciones a seguir ante la presencia de ciertas patologías comunes en las áreas fronterizas, las mismas incluyen las enfermedades enunciadas en el convenio de 1978, y se incorporan otras como dengue, SIDA e inmunoprevenibles. Entre las acciones a desarrollar se destacan: mantener la vigilancia epidemiológica y entomológica en las áreas fronterizas; desarrollar acciones conjuntas a nivel de los programas de vacunación; intercambiar información sobre patología o temas sanitarios de interés para las instituciones de salud de las Provincias Argentinas y Regiones Sanitarias del Paraguay con áreas fronterizas compartidas. Entre otras actividades específicas de cada patología.

Los siguientes ocho artículos hacen referencia a temas como: nutrición y educación alimentaria; formación y adiestramiento de recursos humanos; educación para la salud; saneamiento ambiental; atención hospitalaria; emergencias y catástrofes; vigilancia epidemiológica y organización y desarrollo regional y local.

Por último, el artículo diez establece las disposiciones finales en las que se instituye la creación de un Comité Conjunto de Coordinación, con representación de las cinco provincias argentinas limitrofes con la República del Paraguay que tendrá la función de coordinar y hacer operativo el presente Convenio. Asimismo se establece que tendrá una duración de cinco años.

La principal diferencia entre el convenio de 1978 y 1992 es que en éste último todos tanto en la denominación como en los considerandos, actividades y reglamentaciones dejan explícito que se trata de la salud en la frontera.

En 1997 ambos países a través de sus respectivos Ministerios de Salud firmaron el Plan Operativo de Acciones Conjuntas en Salud en la Región Fronteriza paraguayo-argentina (ARPA) con el objetivo de desarrollar acciones concretas y medidas efectivas en educación para la salud, vigilancia epidemiológica, emprendimientos binacionales y control de la elaboración, tránsito y expendio de alimentos. El ARPA definió niveles de coordinación, seguimiento y evaluación con comisiones técnicas a nivel nacional, regional y de frontera. 
En 2002 se firmó el ARPA II en el cual ambos Ministerios se comprometen a implementar un programa en las áreas de vigilancia epidemiológica y control de enfermedades transmisibles prevalentes, dando prioridad al control del dengue.

En el Protocolo de intenciones entre el Ministerio de Salud de la República Argentina y el Ministerio de Salud Pública y Bienestar Social de la República de Paraguay firmado el 18 de julio de 2006, ratifican "la intención de estimular la cooperación y el intercambio entre los dos países en lo que se refiere a la política de Medicamentos, Estrategias de Atención Primaria de la Salud, Vigilancia y Control de Enfermedades en Zonas Fronterizas, así como a las Políticas de Recursos Humanos en Salud-Medicina Comunitaria", como así también priorizar como temas de interés mutuo, el desarrollo de programas de trabajo e intercambio de experiencias y lecciones aprendidas, en relación a los puntos detallados anteriormente. El presente protocolo tiene una vigencia de dos años renovable automáticamente.

Nuevamente el 4 de diciembre de 2013 el Ministerio de Salud de la República Argentina y el Ministerio de Salud Pública y Bienestar Social de la República de Paraguay firman el Programa Operativo Conjunto de Salud en áreas de frontera, con especial énfasis en la prevención y control del dengue, y el desarrollo de acciones conjuntas de vigilancia epidemiológica y control de otras enfermedades transmisibles prevalentes.

El referido programa será elaborado por los técnicos de las partes y tendrá como objeto la colaboración para el intercambio y formación de recursos humanos, el fortalecimiento de la red local de laboratorios y la aplicación de acciones técnicas orientadas a la promoción de la salud, saneamiento básico, control de enfermedades y a la respuesta de emergencias.

Asimismo, el acuerdo propiciará "el intercambio de información epidemiológica vinculada al seguimiento de enfermedades transmisibles en áreas de frontera promoviendo el uso estratégico de la misma para el desarrollo de acciones de control y mitigación en los niveles locales, departamentales/provinciales".

En 2008 el gobierno de la provincia del Chaco y el gobierno de Paraguay firmaron un convenio de cooperación y colaboración inter-institucional para abordar la problemática sanitaria en el área de frontera compartida. Entre las 
acciones que se prevén está el mantenimiento de la vigilancia epidemiológica en las áreas fronterizas.

Ese mismo año el Gobierno del Departamento de Itapúa y el gobierno de la Provincia de Misiones suscribieron un Convenio Marco de Colaboración y Cooperación cuyo propósito es fortalecer el desarrollo en las áreas de producción, seguridad, turismo, comercio, justicia, derechos humanos y género, medioambiente, salud, energía e infraestructura, cultura y educación.

\section{Acciones de cooperación}

En el marco del Convenio de salud fronteriza que mantiene el Ministerio de Salud de la Argentina y el Ministerio de Salud Pública y Bienestar Social de Paraguay, para el "Control y la Erradicación de Enfermedades Inmunoprevenibles" se han realizado acciones concretas de cooperación entre las que se puede destacar:

En la epidemia de dengue del verano de 2007 que afectó la zona occidental del Paraguay - especialmente la capital - y algunos distritos limítrofes con el Brasil, en donde las manifestaciones del mal adoptaron la forma de "dengue visceral", una nueva modalidad particularmente virulenta de la enfermedad, y constituyó el punto culminante de una cadena previa de brotes clásicos de la enfermedad, que se dieron a lo largo del 2006, la Argentina dio una rápida respuesta a la emergencia, a través del Ministerio de Salud de la República Argentina, que elaboró un Plan de Trabajo conjunto con su par paraguayo. El objetivo fue reforzar el control y la prevención de casos en la frontera común, para lo cual, equipos técnicos del Ministerio de Salud argentino se trasladaron y permanecieron más de 90 dias en las zonas afectadas con el apoyo del Fondo Argentino de Cooperación Horizontal de la Cancillería Argentina (FOAR). El Ministerio de Salud argentino apoyó esta asistencia técnica con vehículos, máquinas pesadas y motomochilas para la fumigación y control de vectores en la zona de frontera (Ministerio de Relaciones Exteriores, Comercio Internacional y Culto, 2008).

Continuando con la asistencia brindada por el Ministerio de Salud de la República Argentina durante la epidemia de dengue del año 2007, expertos en epidemiología de esa cartera se desplazaron en los primeros dias del mes de febrero de 2008 al Paraguay para participar de la campaña de lucha contra la fiebre amarilla. Los mismos recorrieron las zonas infectadas (San Pedro, Laurelty - San Lorenzo), además de colaborar en la sede del Ministerio de Salud Pública y 
Bienestar Social de Paraguay con el equipo responsable de controlar la epidemia (Embajada Argentina en Paraguay, 2008).

Por su parte, el Ministerio de Salud Pública y Bienestar Social de Paraguay, a través de la Resolución $\mathrm{N}^{\circ} 331$, de fecha 21 de noviembre de 2008, dispuso la provisión, en carácter de donación, de 100.000 dosis de vacunas contra la Fiebre Amarilla, así como 20 viales de Gammaglobulina Antirrábica Humana de 300 UI, que habian sido solicitadas por la Titular de Salud de Argentina (Ministerio de Salud Pública y Bienestar Social, 2008).

Además, en abril de 2009, Paraguay entrega a las autoridades sanitarias de Argentina, ocho fumigadoras pesadas, pertenecientes a las reservas del Servicio Nacional de Erradicación de Enfermedades Transmitidas por Vectores (SENEPA), en carácter de préstamo a fin de intensificar los trabajos de control del dengue en las provincias Argentinas (Ministerio de Salud Pública y Bienestar Social, 2009).

En 2010 el Ministerio de Salud de la República Argentina ha realizado una serie de donaciones consistentes en diferentes insumos estratégicos y asistencia técnica a funcionarios paraguayos. Entre los aportes, figura una entrega de más de 5 mil tratamientos antiretrovirales al Ministerio de Salud y Bienestar Social del Paraguay (Embajada Argentina en Paraguay, 2011).

Asimismo, bajo el marco de la Cooperación Horizontal FO-AR del Ministerio de Relaciones Exteriores de Argentina, el Ministerio de Salud ejecuta actualmente proyectos de cooperación bilateral con la República de Paraguay.

Uno de los proyectos de cooperación con resultado exitoso, está relacionado a la Farmacovigilancia. Este proyecto planea actividades donde la Administración Nacional de Medicamentos, Alimentos y Tecnologías (ANMAT) propicia, a través de talleres y otras actividades, capacitación técnica e intercambio de experiencias con su contraparte paraguaya tendientes al fortalecimiento de las capacidades regulatorias en materia de registro y calidad de medicamentos.

A partir de una misión exploratoria de técnicos argentinos de la Administración Nacional de Medicamentos, Alimentos y Tecnología Médica (ANMAT) al Paraguay, en noviembre de 2008, se elaboró un Proyecto conjunto para fortalecer la autoridad de Vigilancia Sanitaria de dicho país. La implementación comenzó en el 2009 con la realización de una serie de talleres y pasantías de formación en ambos países. Primero se capacitó en la Argentina a un total de 81 
funcionarios, de los cuales 16 provenian de dos organismos dependientes del Ministerio de Salud y Bienestar Social de Paraguay: la Dirección Nacional de Vigilancia Sanitaria (DNVS) y el Departamento de Control de Establecimientos de Salud y Afines (DCPYES). Luego se realizaron cursos de capacitación en Paraguay, que permitieron la formación de 65 funcionarios integrantes de distintos organismos relacionados con la temática. Estas actividades contribuyeron a revisar el enfoque de la vigilancia sanitaria y a acordar nuevos procedimientos y criterios de inspección (Ministerio de Relaciones Exteriores y Culto de la Nación Argentina, 2010).

Otro tema de cooperación bilateral con resultados positivos, está vinculado a las acciones para el Fortalecimiento de la Capacidad Institucional del Instituto Nacional de Ablación y Trasplante (INAT) del Ministerio de Salud Pública y Bienestar Social de Paraguay en relación a procuración, ablación y trasplante de órganos y tejidos. El Ministerio de Salud de la República Argentina por medio del INCUCAI esta transfiriendo y dando asistencia técnica a las autoridades sanitarias paraguayas e incluso se está planteando la capacitación de recursos humanos para realizar trasplantes hepáticos en el Paraguay (Embajada Argentina en Paraguay, 2011).

Asimismo, en el marco de la integración regional Argentina y Paraguay participan activamente en el Subgrupo de Trabajo Nro.11 -Salud- de las Comisiones Intergubernamentales de Salud y de las reuniones de Ministros de Salud del MERCOSUR en el Consejo de Salud Suramericano (UNASUR Salud).

\section{COMENTARIOS}

Es importante destacar el notable papel que adquirió la salud, en los últimos años, en la agenda internacional, lo cual abre nuevas posibilidades de cooperación internacional, en tanto que el desarrollo de marcos normativos en las fronteras refleja la importancia de la salud para el comercio, la movilidad de la población, las finanzas y otros temas internacionales.

La cooperación en salud entre Argentina y Paraguay se da tanto en forma bilateral, a través de tratados y convenios entre instituciones gubernamentales de ambos países, como regional, en el marco del MERCOSUR mediante la participación activa en el Subgrupo de Trabajo Nro.11 -Salud- de las Comisiones Intergubernamentales de Salud y de las reuniones de Ministros de Salud que 
tienen lugar en la región; y en el ámbito de La Unión de Naciones Sudamericanas (UNASUR) en el Consejo de Salud Suramericano (UNASUR Salud).

\section{REFERENCIAS}

Convenio Sanitario. (1978). Convenio Sanitario entre el Gobierno de la República Argentina y el Gobierno de la República del Paraguay. Recuperado de: http://trata dos.cancilleria.gob.ar

Embajada Argentina en Paraguay. (2008). Asistencia en ocasión de la epidemia de fiebre amarilla. Recuperado de: http://www.embajada-argentina.org.py/V2/2008/05/ asistencia-en-ocasion-de-la-epidemia-de-fiebre-amarilla/\#more-25

Embajada Argentina en Paraguay. (2011). Asistencia técnica en materia de cooperación. Recuperado de: bilateral. http://www.embajada-argentina.org.py/V2/2011/08/asisten cia-tecnica-en-materia-de-cooperacion-bilateral/\#more-2840

Fogel, R. (2008). La región de la triple frontera: Territorios de integración y desintegración. Sociologías, 10(20).

Frenk, J, y Chacón, F. (1992). Bases conceptuales para la educación e investigación en salud internacional: Salud Internacional, un Debate Norte-Sur. Washington: Organización Panamericana de la Salud. (Serie de Desarrollo de Recursos Humanos No 95).

Maeder, E. J. A. (2010). La frontera argentino-paraguaya; etapas de su delimitación (16181950). Resistencia: Instituto de Investigaciones Geohistóricas (IIGHI-CONICET), Instituto de Historia (UNNE). (Folia Histórica del Nordeste $\left.N^{\circ} 18\right)$.

Mercosur (Mercado Común del Sur). (1991). Acerca del Mercosur. Recuperado de: http:/ / www.mercosur.int/t_generic.jsp?contentid=655\&site=1\&channel=secretaria\&sec cion=2\# social

Ministerio de Relaciones Exteriores y Culto de la Nación Argentina. (2010). Bicentenario de la Patria. Revista FO-AR, 10, (edición especial). Recuperado de: http://cooperacionarg.go b.ar/userfiles/foar-revista-10_1357660737_0.pdf

Ministerio de Relaciones Exteriores, Comercio Internacional y Culto. (2008). Fondo Argentino de Cooperación Horizontal. Revista FO-AR, 9, (edición especial). Recuperado de: http:// cooperacionarg.gob.ar/userfiles/revista-foar-2008b.pdf

Ministerio de Salud Pública y Bienestar Social. (2008). Paraguay dona dosis antiamarílicas y antirrábicas a Argentina. Asunción: Dpto. de Prensa del MSPBS. Recuperado de: 
http://www.mspbs.gov.py/v2/2501-PARAGUAY-DONA-DOSIS-ANTIAMARLICAS-YANTIRRBICAS-A-ARGENTINA

Ministerio de Salud Pública y Bienestar Social. (2009). Lucha contra el Dengue en América del Sur: Paraguay presta maquinas rociadoras a Argentina. Asunción: Dpto. de Prensa del MSPBS. Recuperado de: http://www.mspbs.gov.py/v2/3583-Lucha-contra-el-Dengueen-Amrica-del-Sur-PARAGUAY-PRESTA-MQUINAS-ROCIADORAS-A-ARGENTINA-

Observatorio Mercosur de Sistemas de Salud. (2008). Sitio web: http://www.observasalud.mercosur.int/

Organización Panamericana de la Salud-Organización Mundial de la Salud. (2008) $48^{\circ}$ Consejo Directivo, $60^{a}$ Sesión del Comité Regional, la salud y las relaciones internacionales: Su vinculación con la gestión del desarrollo nacional de la salud, Washington: OPS, OMS. (CD48/15 Esp.)

Organización Panamericana de la Salud. (2007). Salud en las Américas, 2007 (volumen I). Washington: OPS.

Organización Panamericana de la Salud. (2008). $48^{\circ}$ Consejo Directivo, la salud y las relaciones internacionales: Su vinculación con la gestión del desarrollo nacional de la salud. Washington: OPS. Recuperado de http://www.paho.org/spanish/gov/cd/cd4815-s.pdf

Palau, T. (1995). Migraciones limítrofes entre Paraguay y la Argentina: El caso de la provincia de Formosa. Estudios Migratorios Latinoamericanos, 13/14(40-41).

Panisset Ulysses B. (1992). Reflexiones acerca de la salud como asunto internacional: Salud Internacional, un debate Norte-Sur. Washington: Organización Panamericana de la Salud. (Serie de Desarrollo de Recursos Humanos No 95).

Rey Balmaceda, R. (1979). Limites y fronteras de la República Argentina. Buenos Aires: Editorial OIKOS.

Secretaría Administrativa del Mercosur. (2001). Procesamiento de normas en el MERCOSUR el caso SALUD. Recuperado de: http://crics5.bvsalud.org/E/grupos/grupo1/Fraga.pdf

Secretaria del Mercosur. (2012). Normativa seleccionada: Instrumentos fundacionales del Mercosur. Montevideo: Secretaria del Mercosur.

Tobar, S. (2009). Integración en salud desde Mercosur: La salud en los procesos de integración, memorias. Lima: Organismo Andino de Salud, Convenio Hipólito Unanue. 
Tobar, S. (2011). Integración regional y salud: Hacia una mirada de la frontera/The Edmundo Granda Ugalde Leaders in International Health Program. Washington: The Pan American Health Organization.

UNASUR (Unión de Naciones Suramericanas). (2008). Decisión para el establecimiento del consejo de salud suramericano de la UNASUR. Recuperado de: http://www.unasursg.org/documentos-normativos-unasur

UNASUR (Unión de Naciones Suramericanas). (2014). Tratado constitutivo de la unión de naciones suramericanas. Quito: Secretaría General de UNASUR. Recuperado de: http://www.unasursg.org/documentos-normativos-unasur 